\title{
The effect of Pseudomonas aeruginosa on pulmonary function in patients with bronchiectasis
}

\author{
G. Davies*, A.U. Wells", S. Doffman*, S. Watanabe* and R. Wilson*
}

ABSTRACT: Bronchiectasis patients are susceptible to infection with Pseudomonas aeruginosa. Isolation is associated with increased severity of disease, greater airflow obstruction and poorer quality of life. It is not known whether infection by $P$. aeruginosa is a marker of disease severity or contributes to disease progression.

Consecutive non-cystic fibrosis adult bronchiectasis outpatients $(n=163)$ with multiple sputum cultures and follow-up pulmonary function tests were designated, according to isolation of $P$. aeruginosa, as "never infected" (group 1; $n=67$ ), "intermittently isolated" (group 2; $n=82$ ) and "chronically infected" (group 3; $n=14$ ). Based upon change in forced expiratory volume in one second (FEV 1$) \%$ predicted levels at $\geqslant 2$ yrs after presentation, longitudinal behaviour was characterised as "improvement" ( $\geqslant 10 \%$ rise), "decline" ( $\geqslant 10 \%$ fall) or "stability". Baseline pulmonary-function tests and longitudinal behaviour were examined in relation to pseudomonas status.

There was no difference between the groups in age, sex, smoking habit or length of follow-up. Baseline FEV1 levels were highest in group 1 (mean \pm SD: $77.4 \pm 24.3$ ) and higher in group 2 $(67.3 \pm 25.7)$ than in group $3(55.2 \pm 18.5)$. The same significant trends were seen for baseline FEV 1 /forced vital capacity ratios and diffusing capacity of the lung for carbon monoxide levels. Subsequent longitudinal behaviour was linked to baseline FEV 1 levels, which were lowest in patients with improvement and lower in association with decline than with stability. However, longitudinal behaviour did not differ between groups 1, 2 and 3, either before or after adjustment for baseline FEV1 levels.

Infection by Pseudomonas aeruginosa occurs in bronchiectasis patients with more severe impairment of pulmonary function but does not influence rate of decline in pulmonary function either before or after adjustment for baseline disease severity. Thus, Pseudomonas aeruginosa is a marker of bronchiectasis severity but is not linked to an accelerated decline in pulmonary function.

KEYWORDS: Bronchiectasis, Pseudomonas aeruginosa, pulmonary function

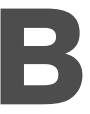
ronchiectasis is defined as chronic dilatation of one or more bronchi. This causes poor mucus clearance and susceptibility to bacterial infection. Once a treatable cause has been excluded, management largely involves physiotherapy and treatment with appropriate antibiotics, for treatment of exacerbations and in some cases prophylaxis. Chronic bacterial infection is common in patients with bronchiectasis, and the bronchial inflammation this stimulates has been implicated in disease progression $[1,2]$. Pseudomonas aeruginosa is an opportunistic pathogen, affecting only those with impaired lung defences, such as patients with cystic fibrosis, other forms of bronchiectasis and severe chronic obstructive pulmonary disease [3-6]. In cystic fibrosis, $P$. aeruginosa infection leads to a deterioration of pulmonary function and ultimately respiratory failure and death $[1,7,8]$. Although $P$. aeruginosa can be isolated intermittently in bronchiectasis, once it becomes a chronic infection it is rarely eradicated, despite intensive intravenous antibiotic therapy $[1,9]$. Chronic infection is associated with more extensive lung disease and more severe airflow obstruction [10], but it is not known whether $P$. aeruginosa is simply a marker of severe disease that has occurred due to another cause or whether it contributes to disease progression.
AFFILIATIONS

*Host Defence Unit, and

\#Interstitial Lung Disease Unit, Royal

Brompton Hospital, London, UK.

\section{CORRESPONDENCE}

R. Wilson

Royal Brompton Hospital

Sydney Street

London SW3 6NP

UK

Fax: 442073518338

E-mail: r.wilson@rbht.nhs.uk

Received:

June 272005

Accepted after revision:

July 312006 
The purpose of the present study was to assess whether $P$. aeruginosa infection in patients with bronchiectasis is associated with a greater rate of decline in pulmonary function.

\section{METHODS}

For the present study, consecutive adult patients from outpatient clinics were included during a 6-month period, who satisfied the following criteria. 1) A diagnosis of bronchiectasis made by high-resolution computed tomography (HRCT). Diagnostic criteria used were: lack of tapering or flaring; and "signet ring" sign. 2) Follow-up at the Royal Brompton Hospital for a period $>2$ yrs. 3) Two or more sputum cultures results available per annum. 4) Two or more pulmonary function tests at a minimum interval of 2 yrs. 5) A negative sweat test to exclude cystic fibrosis. 6) No other major parenchymal lung diseases.

Case notes were reviewed to identify $P$. aeruginosa isolation in sputum cultures. Sputum samples from clinics and hospital stays were processed using selective and non-selective techniques [11]. Patients were designated as: group 1 ("never infected" with $P$. aeruginosa, $\mathrm{n}=67$ ); group 2 ( $P$. aeruginosa isolated at least once, but not on all occasions, "intermittently isolated", $\mathrm{n}=82$ ); group 3 ( $P$. aeruginosa in all cultures, "chronically infected", $n=14$ ). Group 2 included a subgroup of patients developing chronic isolation of $P$. aeruginosa during follow-up $(n=16)$. It is the current authors' practice to perform pulmonary function tests during a period of clinical stability and this was verified in the case notes. This is defined as lack of change in symptoms and no change in treatment, including a requirement for additional antibiotics, for 4 weeks prior to the lung function test. Patients are followed up at least annually in these clinics, whatever their disease severity, so all patients who had appointments in this time were considered. Serial changes between the first and last pulmonary function tests were evaluated.

Forced expiratory volume in one second (FEV1) \% predicted, residual volume (RV) \% pred, diffusing capacity of the lung for carbon monoxide (DL,CO) \% pred, corrected for haemoglobin and FEV1/forced vital capacity (FVC) ratio were noted. FEV1 levels have the strongest correlation with the severity of morphologic abnormalities on HRCT [11, 12] and were designated as the primary end-point. Normal values for pulmonary function testing were validated in the study by CRAPO [13]. Since it is not known whether the rate of decline in bronchiectasis is continuous or whether discrete events, such as infections, lead to irregular periods of decline on a background of general stability, pulmonary function trends were analysed to cover both possibilities. For the primary analysis, based upon clinical experience that deterioration is usually discrete and not continuous: serial change in FEV1 \% pred from baseline to last measurement was characterised as "improvement" (>10\% improvement from baseline value), "decline" (>10\% decline from baseline value) and "stability" (final value within $10 \%$ of baseline value) [12]. A secondary post hoc analysis of serial change in FEV1 expressed as $\mathrm{mL} \cdot \mathrm{yr}^{-1}$ (over the total follow-up time) was also performed.

Patients were managed in the authors' unit by a set protocol, which did not change during the study. Infective exacerbations were treated with oral antibiotics guided by sputum microbiology, antibiotic sensitivities and patient antibiotic history. Failure led to a clinic appointment and admission for i.v. antibiotics if required. Antibiotic prophylaxis was introduced if patients had more than six infective exacerbations per year despite optimal (e.g. physiotherapy) management. Oral antibiotics during the winter months (amoxycillin or doxycycline) for Pseudomonas-negative patients, and nebulised colomycin throughout the year for Pseudomonas patients, were first-line treatments. Asthma and acid reflux were treated in the usual way if present. Inhaled steroids were assessed in an objective manner (lung function and sputum characteristics) and only continued if benefits were demonstrated; oral corticosteroids were only used in severe exacerbations. At the time of the study, long-term macrolide antibiotics were not used as antibiotic prophylaxis.

All routine pulmonary function tests were performed in the authors' dept using the Jaeger Compact Masterlab pulmonary function equipment (Jaeger Ltd, Viasys Healthcare, Hochberg, Germany). Inhaled treatment was not taken on the day of the test. Spirometry was performed in a conventional way by carrying out maximal flow-volume loops in a graphical form of flow versus volume, recorded from a maximal forced expiration, starting from full inspiration, immediately followed by a maximal inspiration; this was performed as one manoeuvre. Several manoeuvres were performed and the results reported are the greatest FEV1 and FVC from at least three technically acceptable manoeuvres, irrespective of the manoeuvre in which they occur, as per recommendations published by the British Thoracic Society and the Association of Respiratory Technicians and Physiologists in 1994 [14]. Jaeger equipment has been used for about $13 \mathrm{yrs}$; although some has been replaced the results obtained have always remained comparable. Biological control tests are performed on a daily basis on normal staff members and no trend regarding a change in pulmonary function test results obtained has been observed for more than a decade. The same predicted values have been used by the authors' dept over the last 12 yrs $[13,15]$.

Population data are expressed as mean $\pm \mathrm{SD}$ for normally distributed variables (age, time between pulmonary function tests, FEV1 \% pred, DL,CO \% pred, FEV1/FVC ratio) and as median values with ranges for abnormally distributed variables (RV \% pred and serial changes in FEV1, expressed as $\left.\mathrm{mL} \cdot \mathrm{yr}^{-1}\right)$. Group comparisons were made using non-paired t-testing or ANOVA for normally distributed variables. Paired t-tests were in group comparisons of serial lung function trends. Wilcoxon's rank sum test or the Kruskal-Wallis test for abnormally distributed variables and Chi-squared statistics for all comparisons of proportion. A p-value of $<0.05$ was taken to indicate statistical significance. Logistic regression models were constructed to determine whether 1) improvement and 2) decline were linked to $P$. aeruginosa status after adjustment for baseline FEV1 levels. This was an important factor, as those who had never isolated $P$. aeruginosa started with a higher FEV1.

\section{RESULTS}

Table 1 compares clinical data between groups 1, 2 and 3; no significant or marginal subgroup differences were identified. 


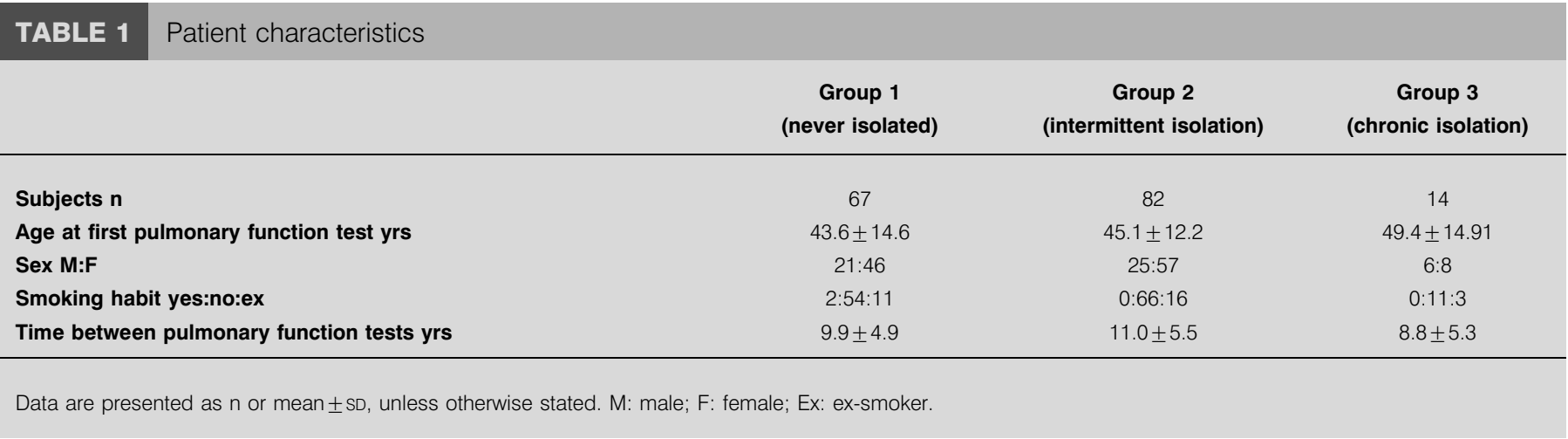

\section{Baseline pulmonary function tests}

As shown in figure 1 and table 2, baseline FEV1 levels were highest in group 1 and higher in group 2 than in group 3 $(\mathrm{p}<0.005)$. Similar trends emerged for FEV1/FVC $(\mathrm{p}=0.02)$ and DL,CO ( $\mathrm{p}=0.02)$, but not for RV (table 2$)$.

Group 2 patients developing chronic isolation of $P$. aeruginosa during follow-up $(n=16)$ were seen for a mean time of $6.34 \pm 3.74$ yrs (range 2.1-21.1 yrs) before $P$. aeruginosa acquisition and for a mean time of $8.75 \pm 5.3$ yrs (range 1.9-11.1 yrs) afterwards. They had lower FEV1 levels at baseline (58.6 \pm 20.5 ; $\mathrm{p}<0.01)$ and higher RV levels $(160.5 \pm 65.8 ; \mathrm{p}<0.05)$ than group 1 patients. However, FEV1 \% pred levels did not differ significantly (paired t-test) before and after the acquisition of chronic $P$. aeruginosa isolation $(58.6 \pm 20.5$ versus $59.4 \pm 25.3)$.

\section{Longitudinal behaviour in relation to baseline FEV 1 levels}

Analysis of changes in FEV1 during follow-up (fig. 2) showed that patients with improvement had the lowest baseline FEV1 levels $(53.7 \pm 20.9)$; FEV1 levels were lower in association with subsequent decline $(71.6 \pm 20.6)$ than with stability $(77.9 \pm 28.7)$. Significance was shown in all comparisons, improvement versus decline $(\mathrm{p}<0.005)$, improvement versus stability $(\mathrm{p}<0.00005)$ and decline versus stability $(\mathrm{p}<0.01)$.

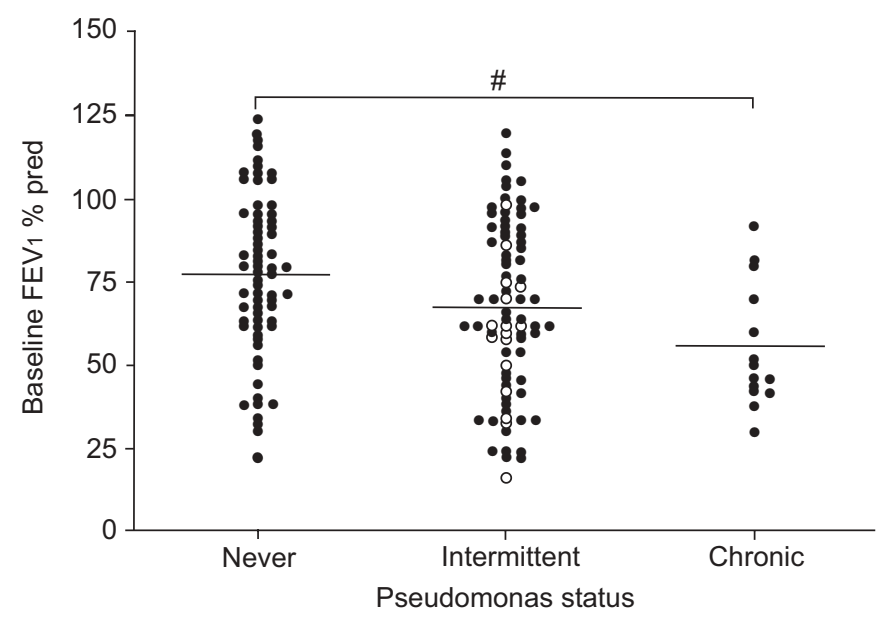

FIGURE 1. Comparison of baseline forced expiratory volume in one second (FEV1) with pseudomonas status. $\bigcirc$ : developed chronic Pseudomonas aeruginosa infection. \% pred: \% predicted. \#: $p<0.005$

\section{Longitudinal behaviour in relation to pseudomonas status}

$P$. aeruginosa status was not linked to longitudinal behaviour. Neither decline (group 1 (21 out of $67,31 \%$ ), group 2 (25 out of $82,30 \%$ ), group $3(5$ out of $14,36 \%$ )) nor improvement (group 1 (13 out of $67,19 \%)$, group 2 (26 out of $82,32 \%$ ), group 3 (3 out of $14,21 \%)$ ) differed significantly between groups. These findings were not altered by analysis of absolute changes in FEV1 per yr (median values: group $1=24 \mathrm{~mL}$; group 2=17 mL; group $3=23 \mathrm{~mL}$ ), with no significant or marginal group differences on non-parametric analysis (Wilcoxon's rank sum test). The $\mathrm{p}$-values for groups 1 versus 2,1 versus 3 and 2 versus 3 are $0.43,0.68$ and 0.92 , respectively. When rate of decline in FEV1 $\left(\mathrm{mL} \cdot \mathrm{yr}^{-1}\right)$ was compared before and after acquisition of chronic $P$. aeruginosa there was no significant difference $(-1.3 \pm 4.3$ versus $0.2 \pm 3.8)$, using Wilcoxon's rank sum test $(\mathrm{p}=0.35)$.

Examination of separate logistic regression models showed that neither decline nor improvement in FEV1 was linked to $P$. aeruginosa status after adjustment for baseline FEV1 status. An improvement of $>10 \%$ in FEV1 was less frequent with a higher baseline FEV1 (odds ratio $(\mathrm{OR})=0.96$; $95 \%$ confidence interval (CI) $0.95-0.98 ; \mathrm{p}<0.0005)$, with no independent relationship with $P$. aeruginosa status $(\mathrm{OR}=0.93 ; 95 \%$ CI $0.51-1.72 ; \mathrm{p}=0.83)$. A decline of $>10 \%$ in FEV1 was related to neither baseline FEV1 nor $P$. aeruginosa status.

\section{DISCUSSION}

A "vicious circle" of bacteria-stimulated, host-mediated lung damage caused by chronic inflammation has been proposed in bronchiectasis [16, 17]. Systemic markers of inflammation are elevated in stable disease and are increased, together with sputum markers, during exacerbations. The level of chronic inflammation is thought to be responsible for disease progression and many of the symptoms that patients experience [18, 19]. Therefore, chronic bacterial infection might be expected to accelerate decline in pulmonary function.

$P$. aeruginosa is an opportunistic pathogen, affecting only those with an impaired host defence. In cystic fibrosis, infection can occur at an early age, before severe bronchiectasis has developed, and various explanations have been put forward for this unique host-bacterial interaction [20-22]. There is an exuberant inflammatory response to the chronic bacterial infection [23] and a large number of exotoxins are produced by $P$. aeruginosa [24]. There is a strong antibody response to $P$. aeruginosa in pulmonary secretions, saliva and serum, and 
TABLE 2 Pulmonary function test results at baseline

\begin{tabular}{lcccc} 
& FEV 1 \% pred & FEV $\mathbf{1}$ FVC & RV \% pred & DL,Co \% pred \\
\hline Group 1 & $77.4 \pm 24.3$ & $69.1 \pm 13.7$ & $120(74-258)$ & $83.4 \pm 13.9$ \\
Group 2 & $67.3 \pm 25.7$ & $63.1 \pm 17.7$ & $126(68-349)$ & $78.8 \pm 20.9$ \\
Group 3 & $55.2 \pm 18.5$ & $58.5 \pm 12.6$ & $141(86-264)$ & $68.9 \pm 22.5$ \\
p-value & $<0.005$ & 0.02 & NS & 0.02 \\
\hline
\end{tabular}

Data are presented as mean $\pm \mathrm{SD}$, with the exception of predicted residual volume (RV) levels, which were positively skewed and are stated as median values with ranges FEV1: forced expiratory volume in one second; FVC: forced vital capacity; DL,CO: diffusing capacity of the lung for carbon monoxide; NS: nonsignificant.

immune complexes are thought to contribute to the inflammatory process [25].

The host-bacterial relationship in non-cystic fibrosis bronchiectasis is less clear. In most cases, the severity of abnormality in the airways is not as great as in cystic fibrosis, and there has been no suggestion of any unique host-bacterial interaction. Intermittent isolation is more common than reported in cystic fibrosis. The change from non-mucoid to mucoid phenotype does occur with chronic infection but is found predominately in patients with severe disease [2]. The isolation of P. aeruginosa in bronchiectasis might be more analogous to chronic obstructive pulmonary disease (COPD) patients, where it has also been recognised as a pathogen in patients with very severe airflow obstruction [26-28]. EvANS et al. [4] showed an association between $P$. aeruginosa and disease severity in bronchiectasis patients. This study showed a significant reduction in FEV1 and FVC in those chronically infected with $P$. aeruginosa compared with those who had never isolated $P$. aeruginosa. An accelerated decline in FEV1 and FVC was also observed in patients with chronic $P$. aeruginosa infection, but the possibility of deterioration prior to $P$. aeruginosa isolation could not be excluded. WeLLS et al. [10] also found an

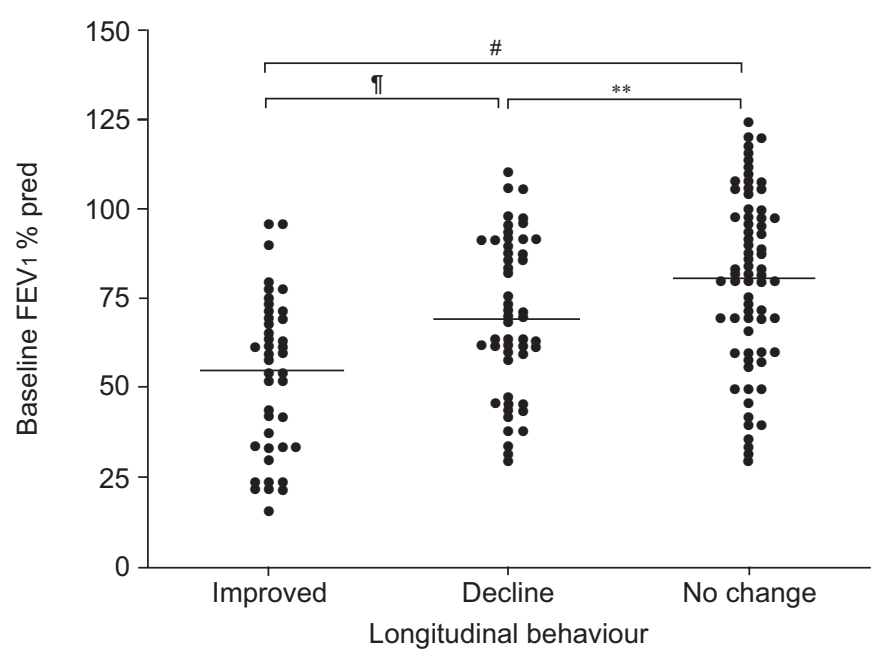

FIGURE 2. Comparison of baseline forced expiratory volume in one second (FEV1) with longitudinal behaviour, when analysed as decline or improvement of $>10 \%$ over time. \% pred: \% predicted. \#: $p<0.00005 ;$ : : $p<0.005 ; * *: p<0.01$. association between colonisation and more severe disease but provided no longitudinal data.

Another study, by MiszKIEL et al. [29], comparing severity of bronchiectasis on thin section HRCT scans with $P$. aeruginosa isolation from concurrent sputum samples, showed a strong relationship between $P$. aeruginosa infection of concurrent sputum samples and increased severity and extensiveness of disease on HRCT. The P. aeruginosa group had more extensive HRCT features of bronchiectasis, a greater degree of bronchial wall thickening and dilatation, as well as evidence of a greater degree of small-airways disease indicated by a more extensive decrease in attenuation.

In a study of 87 patients with non-cystic fibrosis bronchiectasis, in a stable phase of illness, the quality of life of patients infected by $P$. aeruginosa was significantly worse than non- $P$. aeruginosa patients [30]. This paper also showed that the $P$. aeruginosa group had worse pulmonary function, but no significant differences were found between the groups for FEV1 and peak expiratory flow rate unless the length of $P$. aeruginosa infection was considered. Patients infected by $P$. aeruginosa for $>3$ yrs had significantly worse FEV1 $(\mathrm{p}<0.03)$ and bronchiectasis scores $(\mathrm{p}<0.05)$ than those infected with $P$. aeruginosa for less time [30].

An interesting observation in the present study concerns links between baseline FEV1 and longitudinal behaviour. Patients exhibiting improvement or decline had lower baseline FEV1 levels than those with stable disease, who had minor reductions in baseline FEV1 (mean of 77\%). Subsequent improvement was associated with the lowest mean baseline FEV1 (performed in a stable phase of their disease). The present authors believe that this apparent anomaly is probably due to the previous observation [12] that improvement in FEV1 is largely due to clearance of mucus plugging. Therefore, treatment of an underlying condition, e.g. hypogammaglobulinaemia, regular physiotherapy and antibiotic treatment probably led to this improvement in a group of patients in whom major management improvements were attainable. It is important to consider that the lower baseline FEV1 levels in the improvement group provide a larger abnormal pulmonary function signal and therefore a greater opportunity to observe improvement.

In the present study, findings of previous studies that $P$. aeruginosa isolation occurs in some patients intermittently have been confirmed, and since some of these patients were 
followed up for $>10$ yrs it has also been shown that the inevitable progression to chronic infection which occurs in cystic fibrosis does not necessarily occur in non-cystic fibrosis bronchiectasis. There is an association of chronic P. aeruginosa infection with more severe airflow obstruction, which is present in patients who acquired $P$. aeruginosa during the study period and those with chronic infection, but not in the group with intermittent isolation. The association with more severe disease is also true for DL,CO \% pred. However, in bronchiectasis, the severity of the airflow obstruction is not as severe as seen in COPD, where cases usually have FEV1 $<30 \%$ pred. $P$. aeruginosa has a high affinity for mucus, and it is possible that impairment of mucociliary clearance and cough clearance, which occurs in bronchiectatic airways due to mucus hypersecretion, increased mucus viscosity and loss of cilia, predisposes to the colonisation [2]. Another possible factor is antibiotic treatment, which may be given more frequently in bronchiectasis and drive the airway bacterial flora towards the more antibiotic-resistant $P$. aeruginosa $[4,10,30]$.

The present study has not shown any difference in rate of pulmonary function decline between patients with and without $P$. aeruginosa infection. A cohort of patients has also been studied as part of the current research before and after $P$. aeruginosa acquisition, and shows no change in rate of decline in FEV1. These results suggest that $P$. aeruginosa is a marker of disease severity but does not account for the impairment in pulmonary function nor accelerate the decline. Patients were not recruited prospectively with this specific study in mind, and there was therefore no strict protocol for the timing of sputum examination and lung function measurement. However, a protocol for culturing at least once each year and performing lung function every 3 yrs is currently in use. Attempts were made to avoid unrecognised bias by enrolling consecutive patients from the clinic who all had bronchiectasis at the outset. Changes in pulmonary function over time were, in most cases, small and yet there was a high prevalence of both decline and improvement. $P$. aeruginosa does not appear any worse than other species in causing decline. The current authors have previously hypothesised that patients with chronic $P$. aeruginosa infection have a poorer quality of life, in part because they have more severe disease but also because they are given more medication (e.g. nebulised antibiotics) and require more admissions to hospital (because ciprofloxacin is the only available oral antibiotic). It is possible that without this extra treatment patients might have an accelerated decline after $P$. aeruginosa infection. However, the present results show that any accelerated decline after $P$. aeruginosa colonisation can be prevented. Another concern is that progressive lung damage might occur due to $P$. aeruginosa infection without any change in pulmonary function. However, this is unlikely to be clinically important if there has been no change in pulmonary function. This has recently been reported in cystic fibrosis [31]. The current practice of the authors is to only repeat HRCT scans if there is a change in clinical status, because of concerns about the radiation involved.

In conclusion, the present study shows that Pseudomonas aeruginosa status in bronchiectasis is a marker of more severe airflow obstruction but is not associated with an accelerated decline in pulmonary function parameters, even after adjustment for baseline disease severity.

\section{REFERENCES}

1 Rayner CF, Cole PJ, Wilson R. The management of chronic bronchial sepsis due to bronchiectasis. Clin Pulm Med 1994; 1: 348-355.

2 Wilson R. Bronchiectasis. In: Gibson J, Geddes D, Costabel U, Sterk P, Corrin B, eds. Respiratory Medicine. 3rd Edn. Edinburgh, W B Saunders, 2002; pp. 1145-1464.

3 Koch C. Early infection and progression of cystic fibrosis lung disease. Pediatr Pulmonol 2002; 34: 232-236.

4 Evans SA, Turner SM, Bosch BJ, Hardy CC, Woodhead MA. Lung function in bronchiectasis: the influence of Pseudomonas aeruginosa. Eur Respir J 1996; 9: 1601-1604.

5 Monso E, Garcia-Aymerich J, Soler N, et al. Bacterial infection in exacerbated COPD with changes in sputum characteristics. Epidemiol Infect 2003; 131: 799-804.

6 Patel IS, Seemungal TA, Wilks M, Lloyd-Owen SJ, Donaldson GC, Wedzicha JA. Relationship between bacterial colonisation and the frequency, character, and severity of COPD exacerbations. Thorax 2002; 57: 759-764.

7 Fick RB Jr, Sonoda F, Hornick DB. Emergence and persistence of Pseudomonas aeruginosa in the cystic fibrosis airway. Semin Respir Infect 1992; 7: 168-178.

8 Govan JR, Harris GS. Pseudomonas aeruginosa and cystic fibrosis: unusual bacterial adaptation and pathogenesis. Microbiol Sci 1986; 3: 302-308.

9 Wilson R, Cole P. Respiratory tract infections. In: Barnes PJ, ed. Respiratory Medicine - Recent Advances. London, Butterworths, 1993; pp. 95-122.

10 Wells A, Desai S, Whetton C, Wilson R, Cole P. The isolation of Pseudomonas aeruginosa from sputum in idiopathic bronchiectasis: an association with extensive disease and severe airflow obstruction. Am Rev Respir Dis 1993; 147: A645.

11 Roberts HR, Wells AU, Milne DG, et al. Airflow obstruction in bronchiectasis: correlation between computed tomography features and pulmonary function tests. Thorax 2000; 55: 198-204.

12 Sheehan RE, Wells AU, Copley SJ, et al. A comparison of serial computed tomography and functional change in bronchiectasis. Eur Respir J 2002; 20: 581-587.

13 Crapo RO. Pulmonary-function testing. N Engl J Med 1994; 331: 25-30.

14 Guidelines for the measurement of respiratory function. Recommendations of the British Thoracic Society and the Association of Respiratory Technicians and Physiologists. Respir Med 1994; 88: 165-194.

15 Gibson GJ. Standardised lung function testing. Eur Respir J 1993; 6: 155-157.

16 Cole P. A new look at the pathogenesis and management of persistent bronchial sepsis: a vicious circle hypothesis and logical therapeutic connotations. In: Davies RJ, ed. Strategies for the Management of Bronchial Sepsis. Oxford, Medicine Publishing Foundation, 1984; pp. 1-20.

17 Wilson CB, Jones PW, O'Leary CJ, Cole PJ, Wilson R. Validation of the St. George's Respiratory Questionnaire in bronchiectasis. Am J Respir Crit Care Med 1997; 156: 536-541.

18 Stockley RA. Bronchiectasis--new therapeutic approaches based on pathogenesis. Clin Chest Med 1987; 8: 481-494. 
19 Wilson CB, Jones PW, O'Leary CJ, et al. Systemic markers of inflammation in stable bronchiectasis. Eur Respir J 1998; 12: 820-824.

20 Wozniak DJ, Keyser R. : Effects of subinhibitory concentrations of macrolide antibiotics on Pseudomonas aeruginosa. Chest 2004;125: Suppl. 2, 62S-69S; quiz 69S.

21 Hybiske K, Ichikawa JK, Huang V, Lory SJ, Machen TE. Cystic fibrosis airway epithelial cell polarity and bacterial flagellin determine host response to Pseudomonas aeruginosa. Cell Microbiol 2004; 6: 49-63.

22 Aldallal N, McNaughton EE, Manzel LJ, et al. Inflammatory response in airway epithelial cells isolated from patients with cystic fibrosis. Am J Respir Crit Care Med 2002; 166: 1248-1256.

23 Pier GB. Pulmonary disease associated with Pseudomonas aeruginosa in cystic fibrosis: current status of the hostbacterium interaction. J Infect Dis 1985; 151: 575-580.

24 Currie DC, Peters AM, Garbett ND, et al. Indium-111 labelled granulocyte scanning to detect inflammation in the lungs of patients with chronic sputum expectoration. Thorax 1990; 45: 541-544.

25 Hoiby N, Flensborg EW, Beck B, Friis B, Jacobsen SV, Jacobsen L. Pseudomonas aeruginosa infection in cystic fibrosis. Diagnostic and prognostic significance of Pseudomonas aeruginosa precipitins determined by means of crossed immunoelectrophoresis. Scand J Respir Dis 1977; 58: 65-79.

26 Eller J, Ede A, Schaberg T, Niederman MS, Mauch H, Lode $\mathrm{H}$. Infective exacerbations of chronic bronchitis: relation between bacteriologic etiology and lung function. Chest 1998; 113: 1542-1548.

27 Miravitlles M, Espinosa C, Fernandez-Laso E, Martos JA, Maldonado JA, Gallego M. Relationship between bacterial flora in sputum and functional impairment in patients with acute exacerbations of COPD. Study Group of Bacterial Infection in COPD. Chest 1999; 116: 40-46.

28 Soler N, Torres A, Ewig S, et al. Bronchial microbial patterns in severe exacerbations of chronic obstructive pulmonary disease (COPD) requiring mechanical ventilation. Am J Respir Crit Care Med 1998; 157: 1498-1505.

29 Miszkiel KA, Wells AU, Rubens MB, Cole PJ, Hansell DM. Effects of airway infection by Pseudomonas aeruginosa: a computed tomographic study. Thorax 1997; 52: 260-264.

30 Wilson CB, Jones PW, O'Leary CJ, Hansell DM, Cole PJ, Wilson R. Effect of sputum bacteriology on the quality of life of patients with bronchiectasis. Eur Respir J 1997; 10: 1754-1760.

31 de Jong PA, Nakano Y, Lequin MH, et al. Progressive damage on high resolution computed tomography despite stable lung function in cystic fibrosis. Eur Respir J 2004; 23: 93-97. 